\title{
EFEITOS DE FITORREGULADORES E NITRATO DE POTÁSSIO NA GERMINAÇÃO DE SEMENTES DO LIMÃO 'VOLKAMERIANO'
}

\author{
E.O. ONO; S. LEONEL; J.D. RODRIGUES \\ Departamento de Botanica, IB/UNESP, CEP: 18618-000 - Botucatu,SP.
}

RESUMO: Foram estudados os efeitos de substâncias reguladoras de crescimento, bem como do nitrato de potássio $\left(\mathrm{KNO}_{3}\right)$, na germinação de sementes do limão 'volkameriano' (Cïrus volkameriana Pasq.). O experimento foi realizado em germinador, contendo papel de filtro umedecido com água destilada e gerbox, como meio para a germinação das sementes. Estas foram retiradas de frutas maduras, lavadas, secas à sombra e armazenadas durante 101 dias em câmara fria. Logo após, foram tratadas com os fitorreguladores e o $\mathrm{KNO}_{3}$ por 24 horas, conforme os tratamentos: $\mathrm{H}_{2} \mathrm{O} ; 50 \mathrm{mg} / \mathrm{l} \mathrm{de} \mathbf{G A}_{3} ; 250$ mg/l de $\mathbf{G A}_{3} ; 50 \mathrm{mg} / \mathrm{l} \mathrm{de} \mathbf{G A}_{4}+\mathbf{G A}_{7}+$ fenilmetilaminopurina; $100 \mathrm{mg} / \mathrm{l} \mathrm{de} \mathbf{G A}_{4}+\mathbf{G A}_{7}+$ fenilmetilaminopurina; $20 \mathrm{mg} / \mathrm{l}$ de fenilmetilaminopurina; $40 \mathrm{mg} / \mathrm{l}$ de fenilmetilaminopurina e $0,2 \%$ de $\mathrm{KNO}_{3}$. As avaliações foram realizadas depois de 15 dias da semeadura, sendo $\mathrm{o}$ intervalo de 2 dias. Através da análise estatística dos resultados obtidos, foi possível concluir que no tratamento com 50 $\mathrm{mg} / \mathrm{l}$ de $\mathrm{GA}_{4}+\mathrm{GA}_{7}+$ fenilmetilaminopurina houve a maior porcentagem de germinação $(89,00 \%)$ de sementes, num menor tempo médio $(22,29$ dias).

Descritores: Cürus volkameriana, giberelinas, citocininas, nitrato de potássio, germinação de sementes

\section{EFFECTS OF GROWTH REGULATORS AND POTASSIUM NITRATE ON 'VOLKAMERIANA' LEMON SEED GERMINATION}

\begin{abstract}
The purpose of this research was to study the effects of growth regulators and potassium nitrate on 'volkameriana' lemon seed germination. The medium for seed germination was filter paper with distilled water. The seeds were removed from ripe fruits, washed, dried and stored at $4-5^{\circ} \mathrm{C}$. After this the seeds were treated with growth regulators and potassium nitrate during 24 hours. The treatments were $\mathrm{H}_{2} \mathrm{O} ; 50 \mathrm{mg} / \mathrm{lof} \mathrm{GA}_{3} ; 250 \mathrm{mg} / \mathrm{l}$ of $\mathrm{GA}_{3} ; 50 \mathrm{mg} / \mathrm{l}$ of $\mathrm{GA}_{4}+\mathrm{GA}_{7}+$ phenylmethylaminepurine; $100 \mathrm{mg} / \mathrm{l}$ of $\mathrm{GA}_{4}+\mathrm{GA}_{7}+$ phenylmethyla-minepurine; $20 \mathrm{mg} / \mathrm{l}$ of phenylmethylaminepurine; $40 \mathrm{mg} / \mathrm{l}$ of phenylmethylaminepurine and $0,2 \%$ of $\mathrm{KNO}_{3}$. The evaluations were performed every two days, starting 15 days after seeding. The moisture with $\mathbf{G A}_{4}+\mathrm{GA}_{\text {, plus }}$ phenylmethylaminepurine at $50 \mathrm{mg} / \mathrm{l}$ proved to be the most beneficial in enhancing seed germination.

Key Words: Citrus volkameriana, gibberellin, kinetin, potassium nitrate, seed germination.
\end{abstract}

\section{INTRODUÇÃO}

A citricultura $E$ hoje uma importante área de exploração agrícola no Brasil, fazendo com que o país seja o maior produtor de citros e o maior exportador de suco de laranja concentrado do mundo.

Conforme o relatado por TEÓFILO SOBRINHO (1980), a condição essencial para o bom êxito econômico de um pomar comercial de citros, está no plantio de mudas de alta qualidade, isto porque a muda é plantada e cuidada por um período de seis a oito anos, antes de revelar o seu máximo potencial de produtividade e qualidade da fruta (LIMA, 1986).

Segundo COELHO et al. (1983), o tempo de 18 a 36 meses, gasto na formação de uma muda de citros, desde o período de semeadura até o transplante para o local definitivo, é considerado bastante longo, sendo interessante a sua diminuição.

Como forma de acelerar e melhorar a germinação das sementes e também, promover o crescimento das mudas de citros, vários pesquisadores, citados por KAHLON e CHANDLER (1987), preconizam o uso de 
reguladores de crescimento. BURNS e COGGINS (1969), BUTTON et al. (1971) e ACHITUV e MENDEL (1973), recomendam o uso de produtos à base de ácido giberélico, enquanto HAAS e BRUSCA (1954), recomendam o ácido 2,4 diclorofenoxiacético (2,4-D) para o mesmo fím.

Com o trabalho, objetivou estudar-se o efeito de reguladores de crescimento do grupo das giberelinas e citocininas, bem como o do nitrato de potássio $\left(\mathrm{KNO}_{3}\right)$, na promoção e uniformização da gcrminação de sementes do porta-enxerto de limão volkameriano (Citrus volkameriana Pasq.), o qual foi indicado, após trabalhos realizados por SALIBE (1973) e POMPEU JÚNIOR et al. (1976), como um dos porta-enxertos a serem utilizados na citricultura paulista, com o que concorda DORNELLES (1981), o qual também relacionou o Citrus volkameriana, como um excelente porta -enxerto para mandarinas no Rio Grande do Sul.

\section{MATERIAL E MÉTODOS}

O experimento foi conduzido em germinador do tipo FANEN modelo 347-G, pertencente ao Departamento de Botânica do Instituto de Biociências - Campus de Botucatu UNESP. A temperatura no interior do germinador, foi mantida constante a aproximadamente $25^{\circ} \mathrm{C}$ (USBERTI e FELIPE, 1980), e em luz branca constante.

As sementes foram extraídas, manualmente, de frutos maduros, de plantas pertencentes ao pomar da Fazenda Experimental Lageado, no dia 05/05/1992.

Após a extração, as sementes sofreram sucessivas lavagens em água corrente, com a finalidade de retirada da mucilagem. Em seguida, foram colocadas para secar à sombra, durante uma semana (OGATA et al., 1981).

Depois do processo de secagem, as sementes foram armazenadas em câmara fria $\left(4-5^{\circ} \mathrm{C}\right)$, até $14 / 08 / 1992$, quando então foram tratadas com os fitorreguladores e com o nitrato de potássio, durante um tempo de imersão de 24 horas para todos os tratamentos.

As substâncias utilizadas como tratamento, foram as seguintes: a. Pro-Gibb (produto comercial embalado pela Abbott Laboratórios do Brasil Ltda., contendo $\mathrm{GA}_{3}$ à $10 \%$ ); b. Promalin (produto comercial contendo $\mathrm{N}$-(fenilmetil)-1H-purina-6-amina à $1,8 \%$ e $\mathrm{GA}_{4} / \mathrm{GA}_{7}$ à $1,8 \%$, fabricado pela Abbott
Laboratories (U.S.A.); c. Acell (produto comercial contendo $\mathrm{N}$-(fenilmetil)-9-(tetrahidro-2-Hpyran-2yl)-9H-purina-6-amina a $1,3 \%$, fabricado pela Abbott Laboratories (U.S.A.); d. $\mathrm{KNO}_{3}$ (nitrato de potássio-P.A., recomendado pelas Regras para Análise de Sementes (BRASIL, 1976).

Dessa forma, foram realizados os seguintes tratamentos:

. T1 - testemunha, correspondente à imersão em água;

. T2 - $50 \mathrm{mg} / \mathrm{l} \mathrm{de} \mathrm{GA}_{3}$;

. T3 - $250 \mathrm{mg} / \mathrm{l} \mathrm{de} \mathrm{GA}_{3}$;

. T4 - $50 \mathrm{mg} / \mathrm{l}$ de $\mathbf{G A}_{4}+\mathrm{GA}_{7}+$ fenilmetilaminopurina;

. T5 - $100 \mathrm{mg} / \mathrm{l} \mathrm{de} \mathrm{GA}_{4}+\mathrm{GA}_{7}+$ fenilmetilaminopurina;

T6 - $20 \mathrm{mg} / \mathrm{l}$ de fenilmetilaminopurina;

T7 - $40 \mathrm{mg} / \mathrm{l}$ de fenilmetilaminopurina;

T8 - $\mathrm{KNO}_{3}$ (0,2\% P.A.).

Logo após estes tratamentos, as sementes receberam tratamento químico com o fungicida Rodhiauran a 1\% (dissulfeto de tetrametil-tiuram - THIRAM), com a finalidade de prevenir a contaminação por patógenos.

Como meio para a germinação das sementes, empregou-se papel de filtro umedecido com água destilada. Posteriormente, as sementes foram colocadas em gerbox $(11 \times 11 \times 3,5 \mathrm{~cm})$ ja contendo o papel de filtro, sendo que foram colocadas 100 sementes por gerbox, as quais foram umedecidas regularmente.

o delineamento experimental empregado foi o inteiramente casualizado, com 8 tratamentos e 4 repetições, com 100 sementes por parcela.

A contagem da germinação teve início no décimo quinto dia após a semeadura, seguindose leitura constante a cada 2 dias, até o vigésimo quinto dia. Considerou-se como semente germina$\mathrm{da}$, aquela que apresentasse radícula com aproximadamente $2 \mathrm{~mm}$ de comprimento (HADAS, 1976).

Os dados obtidos para porcentagem de germinação, foram submetidos à análise de variância (teste $F$ ), utilizando-se a transformação arc sen da raiz quadrada da porcentagem, sendo as médias comparadas pelo teste Tukey (GOMES, 1966). Além disso, foram realizadas observações quanto ao tempo médio de germinação $(t)$ e velocidade média de germinação (v), os quais foram calculados da seguinte maneira. segundo LABOURIAU (1983): 


$$
t=\Sigma n_{1} \cdot t_{1} / \Sigma n_{1}
$$

e

$$
v=1 / t
$$

onde:

$t=$ tempo médio de germinação (dias);

$\mathrm{n}_{1}=$ número de sementes germinadas num intervalo de tempo:

$v=$ velocidade média de germinação (sem./ dia).

\section{RESULTADOS E DISCUSSÃO}

Através da TABELA 1, pode verificar-se que houve efeito significativo dos tratamentos sobre a germinação de sementes de limão 'volkameriano' (Citrus volkameriana Pasq.). No entanto, não houve significância para tempo médio e velocidade média de germinação.

A observação da TABELA 1 , reflele que o melhor tratamento foi T4 (50 mg/l de $\mathrm{GA}_{4}+$ $\mathrm{GA}_{7}+$ fenilmetilaminopurina), o qual corresponde à mistura de fitorreguladores do grupo das giberelinas e citocininas.

No que se refere à germinação de sementes cítricas, METIVIER (1979) ressalta o papel chave das giberelinas na germinação, estando envolvidas tanto na quebra da dormência como no controle da hidrólise de reservas, da qual depende o embrião em crescimento.

No entanto, além das giberclinas, o mesmo autor relata a presença de outros hormônios envolvidos no processo, como as citocininas, que têm a capacidade de promover germinação em algumas espécies, quebrando a dormência ou causando $o$ início de alguns processos críticos.

Os resultados obtidos, para porcentagem de germinação, mostram que os tratamentos $T 3$ (250 mg/1 de $\mathrm{GA}_{3}$ ) e T5 (100 mg/1 de GA $4+\mathrm{GA}_{7}$ + fenilmetilaminopurina) correspondentes às maiores concentrações de ácido giberélico e ácido giberélico + fenilmetilaminopurina, exerceram efeito contrário à germinação em relação às menores concentrações (T2 e T4), isto é, inibiram o processo.

Portanto, pode concluir-se que as altas concentrações empregadas de GA e GA + fenilmetilaminopurina, aplicadas às sementes, têm influência negativa na porcentagem de sementes germinadas.

Por outro lado, quando se observa o efeito das citocininas na germinação (T6 c T7), é possível verificar que as concentrações empregadas, não foram suficientemente efetivas $\mathrm{cm}$ promover a germinação, bem como em aumentar a sua velocidade, permitindo sugerir que concentrações acima dc $40 \mathrm{ppm}$, poderiam aumentar os valores obtidos.

E importante ressaltar que no estudo realizado, a utilização de fitorreguladores e nitrato de potássio, apresentaram efeito somente na porcentagem de germinação, não influindo no tempo e na velocidade.

CHOUDHARI e CHAKRAWAR (1982), também utilizaram o $\mathrm{KNO}_{3}(1 ; 1,5$ c 2,0\%) para promover a germinação de sementes de limoeiro cravo. Contudo, os autores concluíram que quando comparado com o ácido giberélico, cste tratamento se mostrou ser menos efetivo, o que vem corroborar com os resultados obtidos no presente trabalho.

\section{CONCLUSÃO}

Através dos resultados obtidos. para germinação de sementes de limão 'volkameriano' (Citrus volkameriana Pasq.), tratadas com fitorreguladores c $\mathrm{KNO}_{3}$, concluiu-se que:

- o processo da germinação, levou cm média 38 dias;

- não houve efeito dos fitorreguladores e $\mathrm{KNO}_{3}$ sobre a velocidade média de germinação e tempo médio de germinação;

- a mistura de $50 \mathrm{mg} / \mathrm{l} \mathbf{G A}_{4}+\mathbf{G A}_{7}+$ fenilmetilaminopurina proporcionou $89,00 \%$ dc sementes germinadas em 22,29 dias, correspondendo ao melhor tratamento obtido no experimento. 
TABELA 1 - Porcentagem média de germinação de sementes, tempo médio de germinação (t em dias) e velocidade média de germinação ( $\mathrm{v}$ em sementes/dia) de sementes de limão 'Volkameriano' (Citrus volkameriana Pasq.).

\begin{tabular}{|c|c|c|c|}
\hline \multirow[b]{2}{*}{ Tratamentos } & \multicolumn{3}{|c|}{ Germinação } \\
\hline & Porcentagem & Tempo Médio & Velocidade \\
\hline T1 - testemunha & $80,75 \mathrm{ab}$ & 22,17 a & $0,045 \mathrm{a}$ \\
\hline $\mathrm{T} 2-50 \mathrm{mg} / \mathrm{l} \mathrm{GA}$ & $82,75 \mathrm{ab}$ & 24,11 a & $0,042 \mathrm{a}$ \\
\hline $\mathrm{T} 3-250 \mathrm{mg} / \mathrm{l} \mathrm{GA}$ & $76,00 \mathrm{ab}$ & 23,19 a & $0,044 \mathrm{a}$ \\
\hline $\begin{array}{l}\mathrm{T} 4-50 \mathrm{mg} / \mathrm{l} \mathrm{GA} \mathrm{A}_{4}+\mathrm{GA}_{7}+\text { fenilmetilamino- } \\
\text { purina }\end{array}$ & $89,00 \mathrm{ab}$ & $22,29 \mathrm{a}$ & $0,046 \mathrm{a}$ \\
\hline $\begin{array}{l}\mathrm{T} 5-100 \mathrm{mg} / \mathrm{l} \mathrm{GA} \mathrm{A}_{4}+\mathrm{GA}_{7}+\text { fenilmetilami- } \\
\text { nopurina }\end{array}$ & $85,00 \mathrm{ab}$ & 24,30 a & $0,042 \mathrm{a}$ \\
\hline T6 - $20 \mathrm{mg} / \mathrm{l}$ fenilmetilaminopurina & $68,50 \mathrm{~b}$ & $23,40 \mathrm{a}$ & $0,043 \mathrm{a}$ \\
\hline $\mathrm{T} 7$ - $40 \mathrm{mg} / \mathrm{l}$ fenilmetilaminopurina & $74,25 \mathrm{ab}$ & 25,01 a & $0,040 \mathrm{a}$ \\
\hline $\mathrm{T} 8-\mathrm{KNO}_{3} 0,2 \%$ & $73,00 \mathrm{ab}$ & $22,77 a$ & 0,045 a \\
\hline
\end{tabular}

Médias seguidas pelà mesma letra não diferem entre si, pelo teste de Tukey $(\mathbf{P}<0,05)$.

\section{REFERÊNCIAS BIBLIOGRÁFICAS}

ACHITUV, M., MENDEL, K. Effect of certain treatments on the germination of sweet lime (Citrus limettioides Tan.) seed. Plant Propagation, v.19, n.4, p.15-20, 1973.

BRASIL, Ministério da Agricultura. Regras para análise de Sementes. Brasília, 1976. 176p.

BURNS, R.M., COGGINS JR., C.W. Sweet orange germination and growth aided by water and gibberellin seed soak. California Agricultural, Oakland, v.23, n.12, p.18-19, 1969.

BUTTON, J., BORMAN, C.H., HACKLAND, B.A. Effect of presowing treatments on the germination of Poncirus trifoliata and troyer citrange seeds. Citrus and subtropical fruit Journal, Schagn, v.451, p.9-11, 1971 .

CHOUDHARI, B.K., CHAKRAWAR, V.R. Effect of seed treatment using some chemicals on the shoot and root growth of Rangpurlime (Citrus limonia Osbeck). Journal of Maharashtra Universities, Parbhani, v.7, n.1, p.66-68, 1982 .

Sci. agric., Piracicaba, 50(3):338-342, out./dez., 1993
COElHo, Y. da S., OlIVEIRA, A.A.R., CALDAS, R.C. Efeitos do ácido giberélico (AG3) no crescimento de porta-enxertos para citros. Pesquisa Agropecuária Brasileira, Brasília, v.18, n.11, p.1229-1232, 1983.

DORNELLES, C.M.M. Porta-enxerto para mandarinas (Citrus deliciosa Tenore) no Rio Grande do Sul. In: CONGRESSO BRASILEIRO DE FRUTICULTURA, 6., Recife. 1981. Anais ... Recife: Sociedade Brasileira de Fruticultura, 1981. p. $702-708$.

GOMES, F.P. Curso de estatística experimental. São Paulo: Nobel, 1966. 404p.

HAAS, A.R.C., BRUSCA, J.N. 2,4-D treatment of citrus seeds. California Agricultural, Oakland, v.8, n.2, p.8-12, 1954 .

HADAS, A. Water uptake and germination of leguminous seeds under changing external water potential in osmotic solution. Journal of Experimental Botany, Oxford, v.27, p.480-489, 1976. 
KAHLON, P.S., CHANDLER, D. A study on the seed germination and subsequent seedling growth in peach (Prunus persica Batsch) cv Shasbati. Research and Development Reporter, Amritsar, v.4, n.1, p.81-84, 1987.

LABOURIAU, L.G. A germinação de sementes. Washington: Organização dos Estados Americanos, 1983. $174 \mathrm{p}$.

LIMA, J.E. de O. Novas técnicas de produção de mudas cítricas. Laranja, Cordeirópolis, v.2, n.7, p.463-468, 1986.

METIVIER, J.R. Citocininas e giberelinas. In: FERRI, M.G. Fisiologia vegetal. São Paulo: EDUSP, 1979. v.2, p.93-162.

OGATA, T., SOUZA, M. de, SANTOS, M. da G.F.M. Poliembrionia, efeitos do nitrato de potássio e da permanência de sementes no germinador, na separação e germinação dos embriôes de citros. In: CONGRESSO BRASILEIRO DE FRUTICULTURA, 6, Recife, 1981 . Anais ... Recife: Sociedade Brasileira de Fruticultura, 1981 p.693-701.
POMPEU JÚNIOR, J., FIGUEIREDO, J.O. de, RODRIGUEZ, O. Porta-enxertos para laranjeira Valência, clone nucelar, em solo arenoso. In: CONGRESSO BRASILEIRO DE FRUTICULTURA, 1, Itaguaí, 1975. Anais ... ltaguaí: Sociedade Brasileira de Fruticultura, 1976, p.371-379.

SALIBE, A.A. Comportamento do Citrus volkameriana Pasq. como porta-enxerto para citros. In: CONGRESSO BRASILEIRO DE FRUTICULTURA, 1, Campinas, 1971. Anais ... Campinas: Sociedade Brasileira de Fruticultura, 1973. p.367-373.

TEÓFILO SOBRINHO, J. Propagação dos citros. In: RODRIGUES, O., VIÉGAS, F., POMPEU JÚNIOR, J., AMARO, A.A. Citricultura brasileira. 2.ed. Campinas: Fundaçāo Cargill, 1980. v.1, p.299-318.

USBERTI, R., FELIPE, G.M. Viabilidade de sementes de Citrus limonia Osb. com baixo teor de umidade, armazenada em diferentes temperaturas. Pesquisa Agropecuária Brasileira, Brasília, v.15, n.4, p.393-397, 1980.

Enviado para publicação em 18.05 .93

Aceito para publicaçāo em 15.07 .93 